\title{
Interpersonal dynamics in sport: The role of artificial neural networks and 3-D analysis
}

\author{
PEDRO PASSOS \\ Technical University of Lisbon, Lisbon, Portugal \\ and Lusófona University of Humanities and Technologies, Lisbon, Portugal \\ DUARTE ARAÚJO \\ Technical University of Lisbon, Lisbon, Portugal \\ KEITH DAVIDS \\ University of Otago, Dunedin, New Zealand \\ LUÍS GOUVEIA \\ University of Lisbon, Lisbon, Portugal \\ and \\ SIDÓNIO SERPA \\ Technical University of Lisbon, Lisbon, Portugal
}

\begin{abstract}
In previous attempts to identify dynamical systems properties in patterns of play in team sports, only 2-D analysis methods have been used, implying that the plane of motion must be preselected and that movements out of the chosen plane are ignored. In the present study, we examined the usefulness of 3-D methods of analysis for establishing the presence of dynamical systems properties, such as phase transitions and symmetry-breaking processes in the team sport of rugby. Artificial neural networks (ANNs) were employed to reconstruct the 3-D performance space in a typical one-versus-one subphase of rugby. Results confirm that ANNs are reliable tools for reconstructing a 3-D performance space and may be instrumental in identifying pattern formation in team sports generally.
\end{abstract}

Identifying the reasons for which some individuals and teams achieve success is one of the most common goals in the research literature on sport performance. The methodologies used to study sport performance have been established to compare the actions of successful and unsuccessful teams and athletes, and the outcomes include a range of statistical data of discrete actions performed by teams and players during competition (see Hughes \& Franks, 2004). Despite the importance of these data, a significant criticism of the notational analysis of sport performance is that it does not identify the reasons for those discrete actions in order to explain the difference between successful and unsucessful teams. The data typically inform us of what happens, but not of how and why it happens.

To understand the dynamical processes in sport performance, McGarry and Perl (2004) used a specific type of neural network: the Kohonen feature map. They argued that the neural networks approach must be used first to recognize situations and analyze processes, and only then to identify decision-making processes. However, with that

Correspondence concerning this article should be addressed to P. Passos, Rua Josefa Galbis Diniz, No. 153, 2. Dt., Arneiro, 2775-522 Carcavelos, Portugal (e-mail: p.passos@clix.pt). approach, a 2-D information structure (e.g., a pair of coordinates) was implemented to represent a pattern, which decreases the ability of a neural network to analyze the complex processes that emerge in sports contests.

Additional problems of network learning processes include their dependency on input patterns and the fact that those patterns change continuously as a result of a player's tactical behaviors. A theoretical rationale from the field of ecological psychology allows us to understand that tactical behaviors are dependent on the information available in specific contexts, and that that information is geared to each player's tactical behavior. Clearly, the use of Kohonen feature maps to study performance might incur problems in reliably recognizing patterns in sports contests. Another method proposed by McGarry and Perl (2004) is the dynamical controlled network (DyCoN), already tested successfully in different areas of sport (Perl, 2002). This method deals with the problem of dependency on input patterns. The DyCoN has the ability to learn continuously or in discrete phases, so input patterns need not be presented to the network in a single data set. McGarry and Perl (2004, p. 241) argued that the information acquired during the learning of the $\mathrm{DyCoN}$ could be used in "complementing our understanding of the processes that take place in the learning of cognitive and/or motor skills 
in humans." This proposal raises a paradox: How can sports contests be interpreted as dynamical systems with an applied methodological mode of functioning based on discrete cognitive approaches?

This question highlights the need for a methodology that will allow researchers to analyze the complexity of dynamical processes in sport performance when they seek to establish parameters in the three planes of motion. The artificial neural network (ANN) proposed in this article represents an attempt to initiate this methodological approach.

\section{Previous Research on Team Sport Subphases}

Viewed as dynamical systems, team ball sports are highly complex phenomena because of the number of degrees of freedom involved that characterize the relationships between system components that evolve over time (e.g., an attacker and a defender are the two components of a dyadic system). Therefore, interpersonal coordination within teams emerges from the coupling of the components of the system (i.e., the players). The first step in understanding team ball games as dynamical systems is to find simple measures that capture the complexity of the system. To achieve this goal, the parameters that best describe interpersonal coordination over time need to be discovered.

Some initial attempts have been made to develop tools for collecting such data from real game situations, thereby allowing an analysis that goes beyond the use of central tendency parameters, which provide only intermittent data. For example, within the framework of dynamical systems theory, Araújo, Davids, Saínhas, and Fernandes (2002) conceptualized the attacker-defender dyad in basketball as an interpersonal coordination system, suggesting that the attacker and the defender are two elements of a complex, dynamical system linked by visual (and other) informational fields.

In their study, Araújo et al. (2002) used a methodology to measure the (2-D) horizontal-plane trajectory of the center of mass of each player. Results were reported in a qualitative analysis substantiated by position-time (e.g., interpersonal distance over time, distance of each player to basket over time) and position-position (e.g., defender position as a function of attacker position) coordinates. ${ }^{1}$ Data supported the view that a symmetry-breaking process had occurred in successful dribbling when there was an abrupt change in the shape of the curve describing the interpersonal distance of each attacker-defender dyad.

Under the distinct task constraints of rugby, many similar instances of interpersonal coordination may be observedfor example, attacker-versus-defender movement patterns near the try line. Here, the goal of the attacker is to cross the try line and ground the ball by hand. The defender attempts to remain between the attacker and the try line by counterbalancing any movement of the attacker, resulting in a state of interpersonal coordination during this subphase of the game. As previous work on basketball (Araújo, Davids, Bennett, Button, \& Chapman, 2004) suggests, in rugby the attacker's decision making should emerge from his or her movements during a process of searching for an available running line toward the try area and avoiding a tackle. During a successful run by the attacker, the initial state of order of the dyad (e.g., a positional balance between attacker and defender) is annihilated or sustained by information fields. A new state of order emerges in this dynamical system during a phase transition.

As we stated earlier, in exploring the emergence of symmetry-breaking processes in team ball sports, Araújo et al. (2004; Araújo et al., 2002) used 2-D analysis techniques. However, 2-D analysis methods require that the plane of motion of each participant be preselected, and, therefore, movements out of the chosen plane are ignored (Bartlett, 1997). Moreover, in qualitative terms, Kelso, Buchanan, DeGuzman, and Ding (1993) demonstrated that when motion is restricted to the horizontal plane, only the symmetric and asymmetric modes of coordination are stable over a range of frequencies. For frequencies above that range, however, no comparably stable pattern is available in the horizontal plane of motion. The system consisting of an established, active set of components that have been self-organized for particular movement patterns is no longer able to support such behavior. To achieve stability, previously quiescent system degrees of freedom (i.e., those in the vertical dimension) are spontaneously recruited. With the availability of these additional degrees of freedom, the system can attain stability once more. It is noteworthy that the new movement pattern may still be topologically equivalent to the previous one, but additional degrees of freedom are required to perform the task. Consequently, although Araújo et al. (2004; Araújo et al., 2002) could observe phase transitions in interpersonal coordination dynamics only in the horizontal plane, symmetry-breaking processes might also be observable in the vertical plane.

In methodological terms, according to Bartlett (1997), the major advantages of 3-D motion analysis over 2-D analysis include the facts that it (1) can show the complete spatial motion of participants; (2) allows other angles of vision - for example, the trajectories of attackers and defenders can be visualized from above; and (3) enables the reconstruction of simulated views of performance other than those seen by the cameras. Therefore, with 3-D analysis, players' displacement during subphases of games can be observed from any chosen angle, and analysis of symmetry-breaking processes is not limited as it is with the use of only a horizontal-plane analysis.

\section{Building and Developing a 3-D Model}

To analyze the trajectory of both players in a dyad, we used a 3-D image measurement technique called videogrammetry (Gruen, 1997). The goal of this technique is to reconstruct 3-D space by transforming the pairs of coordinates of each camera into three coordinate planes $(x, y$, and $z$ ). The reconstruction of 3-D space is termed the resolution stereo problem, and the technique that offers the most advantages is the ANN approach (Memon \& Khan, 2001).

The advantages of adopting ANN to solve the resolution stereo problem include the following: (1) One can dispense with the knowledge of intrinsic camera param- 
eters demanded by linear models (e.g., focal distance, image center, image scale, distort coefficient lens), as well as extrinsic camera parameters (e.g., camera position related to origin, camera orientation). (2) With the ANN, it is possible to work with any kind of camera. (3) There is no need for cameras to be in a fixed location in relation to the origin. (4) The axes among the cameras do not need to be aligned in a fixed position. (5) Unlike linear models, the ANN deals automatically with the distortion lens problem (Gruen, 1997).

An ANN is an information-processing system with parallel distribution and a tendency to store experimental data for future use (Haykin, 1994; Smith, 2001; Stergiou \& Siganos, 1996). The ANN's functions are based on two main facts: (1) that knowledge is acquired through a learning process and (2) that the interneuron connection strengthens with synaptic weights that are used to channel information (Haykin, 1994). In our study, we used an ANN of the feed-forward class because it has a topology that allows information to flow from input to output.

Finally, using ANN enhances the coherence between the theoretical and methodological approaches we are following. As an example, the ANN can deal with the variability of camera parameters, whereas linear methods cannot. From a theoretical perspective, dynamical systems theory assumes that both linear and nonlinear processes exist in biological movement systems (see, e.g., Kelso, 1995); from a methodological point of view, using linear methods biases the analysis toward linearity, thus creating a fundamental contradiction.

To demonstrate how ANNs might be a useful methodological advance in the study of pattern formation and interpersonal dynamics in team sports, which are viewed as complex systems, we applied the method for analyzing phase transitions in rugby. Rugby is a performance context characterized by intense physical contact. According to the rules of the game, defenders are allowed to tackle attackers, take them to the ground, and recover possession of the ball for their team. Moreover, it is not permissible to pass the ball forward with the hands, and the ball's shape makes it difficult to control it with the feet. These task constraints characterize rugby as a sport with a different functional organization than that of the sport of basketball, investigated in previous work.

The rugby coaching literature provides detailed descriptions of optimal ways to tackle an opponent (see, e.g., Biscombe \& Drewett, 1998). Those descriptions explain what defenders must do when contact with attackers occurs. However, contributors to the pedagogical literature typically fail to address how defenders can drive attackers to a contact point. Perhaps the exception is Greenwood (2003), who stated that for a successful tackle, defenders must "start fast but arrive in balance" (p. 166). We can speculate from his words that if defenders "arrive in balance," the dyad formed between an attacker and a defender would maintain its initial state of order, and that if defenders cannot "arrive in balance," a symmetry-breaking process is more likely to occur. However, the term "arriving in balance" is rather nebulous for understanding the emergence of stabilities and instabilities in the interpersonal dynamics of team ball games. A particularly interesting question concerns the parameters in the attacker-defender dyads that enable them to maintain or change their functional organization.

Thus, our study had three goals, all of which we achieved using subphases of the sport of rugby: (1) to identify phase transitions in a team ball sport with task constraints different from those of basketball, (2) to present a 3-D analysis of interpersonal dynamics of attacker-defender dyads, and (3) to identify parameters to measure dynamical systems properties in these dyads.

\section{METHOD}

\section{Participants}

Eight male rugby players (11-12 years of age) participated in the study (mean years of rugby practice $=4.0, S D=0.5$ ). The decision to study such young players was made to prevent the confounding effects of expertise and different degrees of learning, since we believed that these variables would have biased the data (cf. Zanone \& Kelso, 1994). We followed the procedures outlined by Bates, Dufek, and Davis (1992) for ensuring a correspondence between the number of trials (task repetitions) and the number of participants: For sample sizes of 10,5 , and 3 trials, the sample sizes of participants should be 5,10 , and 20 , respectively. Because of the nature of our task, participant interactions were analyzed as events of interpersonal coordination in attacker-defender dyads. Therefore, we randomly allocated a number to each rugby player of our sample. Each player acted as both attacker and defender and participated with every other player in dyads. In order to prevent possible fatigue effects on performance, it was decided that each dyad would perform two trials. With all possible number combinations, we observed the responses of 32 different dyads.

\section{Task}

We designed a task that was representative of a subphase of rugby involving the minimum number of players: the common one-versusone situation near the try line. In this subphase, a pair of rugby players with opposing functions and goals forms a dyad in which the attacker aims to place the ball in the try area with his hand and the defender aims to stop the attacker's progression toward the try line and take him to the ground within the rules of the game. We established the depth and width of the field following the procedures of Biscombe and Drewett (1998), who recommended the field dimensions for one-versus-one subphase practice tasks. This advice helped us to construct the task constraints of the experimental work in our study (see Figure 1).

According to their coaches, all the selected participants knew how to tackle (i.e., how to intercept an attacker and stop his progression toward the try line). Defenders attempted to contact attackers with the shoulders and arms, and they held their legs or trunks to take them to the ground (Biscombe \& Drewett, 1998).

\section{Instruments}

Player motion was captured by two digital video cameras. For image treatment, we used the software TACTO 7.0, digitized at 25 frames/sec (Fernandes \& Caixinha, 2003). The ball used was size 5, as required by the Portuguese Federation of Rugby for the age group of the participants. Twelve rods, each $1.8 \mathrm{~m}$ in length, were used to build a cube-shaped reference structure, which was moved in order to cover the entire data collection volume. This reference structure gave us the points used for the training and validation procedures for the ANN. 


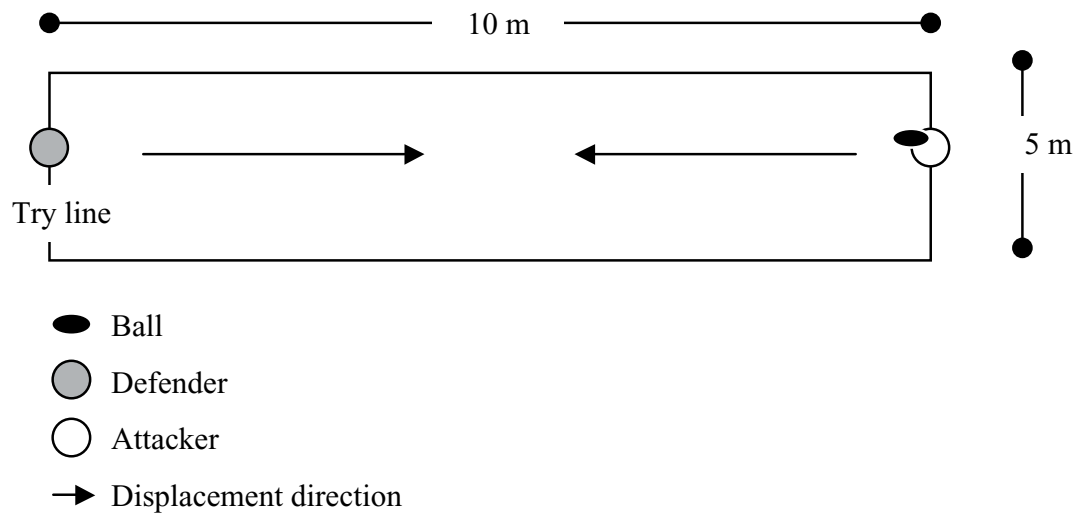

Figure 1. Task design.

\section{Procedures for Recording the Dyad's Action}

Camera positions. Bartlett (1997) suggested that the angle between cameras for motion capture should vary from $60^{\circ}$ to $120^{\circ}$. We followed this guideline even though the ANN procedures did not require it, as we explained earlier. To synchronize the images recorded by both cameras over trials, we used the specific frame in which the attacker touched the ball with the foot before initiating his attempt to cross the try line. The video images were acquired by the computer via USB, using Pinnacle Studio version 8.0 SE software, and saved as AVI files.

Image processing. To extract data on player displacement coordinates, we used TACTO 7.0 (Fernandes \& Caixinha, 2003). The procedure consisted of following with the mouse the working point located in the middle of the abdominal area of each player, after which the coordinates were automatically extracted.

As noted earlier, the ANN architecture used in the present research program belonged to the feed-forward class. It consisted of four input neurons, corresponding to four respective coordinates of stereo images for each player (i.e., two from the frontal camera [C1x, $\mathrm{C} 1 y]$ and two from the transverse camera $[\mathrm{C} 2 x, \mathrm{C} 2 y])$; six neurons in a hidden layer; and three output neurons, corresponding to the real coordinates $x, y$, and $z$ (Memon \& Khan, 2001; see Figure 2).

\section{ANN Training and Validation}

To ensure accurate information running on the ANN, a previous stage of training is required. The ANN training procedure allowed us to convert the known stimulus (i.e., point coordinates from two images) to an adequate response (i.e., spatial coordinates of that point).

The procedure consisted of using a backpropagation algorithm to minimize the difference between the actual output and the desired output (Haykin, 1994). The backpropagation process had two phases: (1) propagation of the functional signal, signifying that information flowed in a positive direction, from input to output, layer by layer, while the synaptic weights were maintained; and (2) estimation of the errors (e.g., difference between the actual output and the desired output). Those errors were then backpropagated to the network in the opposite direction - from output to input - and the synaptic weights were adjusted to reduce errors to acceptable values.

In the learning network, we used a backpropagation algorithm, which is an iterative process indicating that there is an iterative change in the parameters of the transfer function of each neuron in the network. This change was made after the network was fed the 2-D coordinates of both cameras and the output was compared against the actual coordinates for the corresponding point. Using this procedure, it was necessary to know the actual coordinates of a set of points from the space where a particular task was performed.

Those points were extracted from the cube-shaped reference structure described earlier, which we moved in order to cover the entire surface/volume of the performance field. This structure is similar to that suggested by D'Appuzo (2002), who used it to measure moving surfaces and track body parts (e.g., left wrist) before transforming the data to a 3-D shape to capture motion information. The reference structure was composed of 330 points, 108 of which were used in the training procedure and the remainder used to validate the network.

The latter procedure consisted of feeding the network a set of pairs of 2-D coordinates (extracted from the reference structure but not used in the training stage) and comparing the ANN outputs against the spatial coordinates actually observed. The agreement of these values enabled confirmation of the accuracy of the ANN tool.

\section{Network Reliability}

Accuracy. Accuracy is a basic concept that needs to be measured in order to evaluate network performance. Gruen (1997, p. 167) referred to accuracy as the quality of a model whereby the estimated coordinates of a given point in the model correspond to the actual coordinates of the same point. According to Memon and Khan (2001), model accuracy should be assessed by comparing the predicted coordinates of a set of points not used in the training procedure against coordinates that are actually observed. The difference between the predicted and actual coordinates can be used to calculate the mean error value for the network, providing a measure of accuracy. The lower the mean error value, the more accurate is the network. The average root mean square error, which reflects the mean difference between actual and predicted 3-D coordinates (reflecting system inaccuracy) calculated over 110 points (not used in the training procedure), had a value of $1.3 \mathrm{~cm}$, which is acceptable for the stated purpose. This value is small enough to avoid a measurement bias in coordinate estimation.

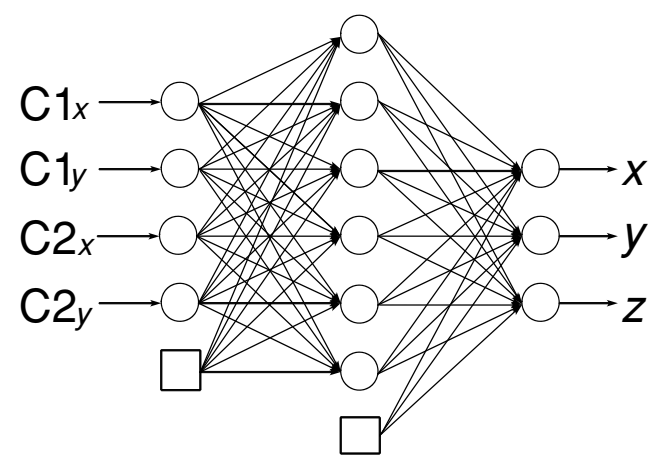

Figure 2. Artificial neural network architecture. 
Precision. Precision can be defined as the closeness of the predicted coordinates when the same actual point is digitized (in both video streams) several times. Ideally, the predicted coordinates would be equal every time a particular point is digitized. However, some aspects contribute to small differences among them, including (1) the function of transferring coordinates from 2-D to 3-D, (2) the quality of the software used in the digitizing process, (3) the skill of the experimenter in manipulating the pointing device (e.g., the computer mouse), (4) the resolution of the video streams, and (5) the positioning of the video cameras.

Precision is commonly operationalized as the $S D$ of a set of predicted 3-D coordinates for each particular digitized point. Since constant precision could not be guaranteed a priori over the entire playing field, we used eight points (digitized six times in random order) located at the extremes of the field in order to quantify a pooled $S D$, which is a more robust estimate of overall precision. The obtained pooled $S D$ was $0.03 \mathrm{~m}$, which is an acceptable figure because it corresponds to $0.3 \%$ of the playing field length.

\section{Measurement of Dependent Variables}

The following dependent variables were calculated in the study.

Distance of attacker and defender from the try line. This variable provides a measure of the absolute distance of each player from the try line, plotted over time. This variable was measured by calculating the distance (along a straight line) between the closest point of the try line and each player. It was calibrated in centimeters and displayed in 2-D position-time graphics. This variable is also calibrated in meters and displayed in 3-D graphics.

Distance of attacker and defender to both lateral lines. This variable provides a measure of the absolute distance of each player from both lateral lines. It was calibrated in meters and displayed in 3-D graphics.

Vertical oscillation. This variable provides a measure of the height (in centimeters) of the working point (located in the middle of the abdominal area of each participant in the dyad) to the ground over time. It was displayed in 3-D graphics.

Stability of vertical oscillation. This variable provides a measure of the means and $S D$ s of the height of the working point (in meters) over time.

\section{RESULTS}

We begin this section by presenting the results of the phase transition analysis with 2-D position-time graphics, to ascertain whether or not the same patterns identified in basketball (see Araújo et al., 2002) exist in the performance context of rugby. We follow that analysis with a presentation of 3-D graphics in order to identify phase transitions that could be hidden within the 2-D positiontime graphics. The 3-D graphical analysis also helped us to identify parameters (e.g., vertical oscillation) that could be the source of differences between a defender's supremacy (a successful tackle) and an attacker's supremacy (a scored try). Finally, we present a stability analysis to show how the vertical oscillation parameter behaved in successful tackles and in try situations.

\section{Phase Transition Analysis}

In Figure 3, two main features of the data may be observed. For successful tackles (Figure 3A), there is no crossover of player displacement trajectory lines. Despite the variability of attacker behavior, both lines of displacement maintained the same pattern over time, with the defender always remaining closer to the try line as the initial state of order of the dyad was maintained.

For try situations (Figure 3B), it can be observed that, initially, both displacement trajectories displayed a pattern similar to those of successful tackles by the defender. However, over time the attacker's trajectory became more adjacent to that of the defender, and suddenly a crossover occurred. The attacker became the player closer to the try line, and the initial state of order of the dyad was annihilated, with a new state of order emerging.

\section{3-D Analysis}

With the lateral graphics (Figure 4), two kinds of analysis were conducted. We searched for parameters that could be used to describe motion differences between try situations and sucessful tackles resulting from the defender's successfully preventing a try. Our analysis shows that the variables of distance to try area ( $y$ values) and vertical oscillation ( $z$ values) are the sources of two main differences between those situations. Defenders were usually more advanced in the field when a try was scored than when a successful tackle was made (for successful tackles, de-
A

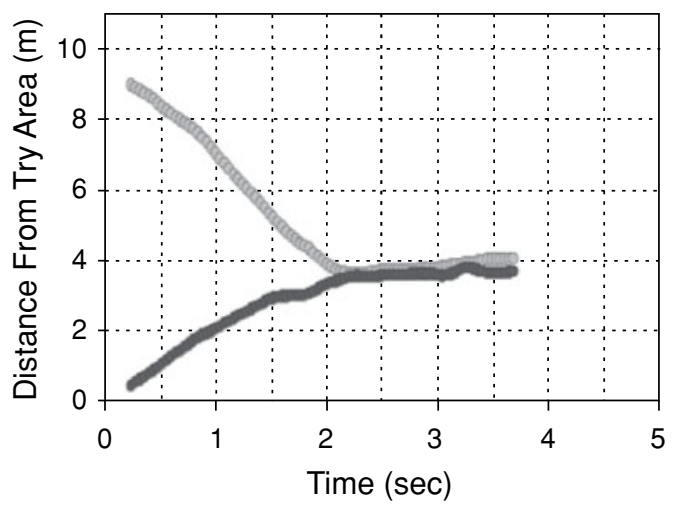

B

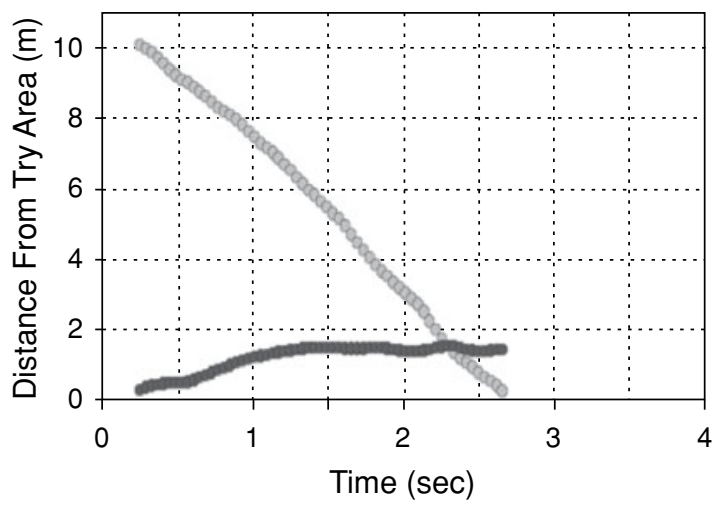

Figure 3. Distance from try area (in meters) over time (in seconds) of the attacker (gray line) and the defender (black line). (A) Successful tackles. (B) Tries. 

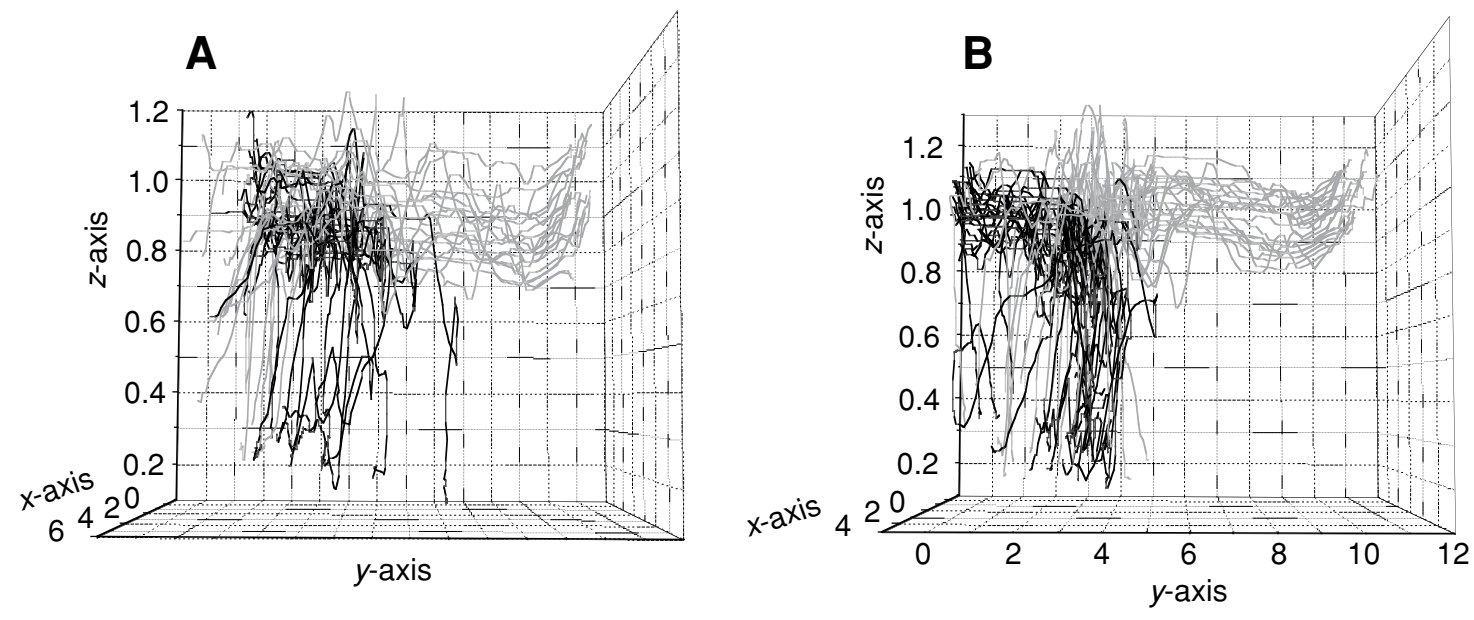

Defender Running Direction

Figure 4. Distance from try area ( $y$ values, in meters) of the attacker (gray lines) and the defender (black lines), and vertical oscillation $(z$ values, in meters). (A) Tries $(n=21)$. (B) Successful tackles $(n=26)$.

fenders acquired a position in the field 2-4 $\mathrm{m}$ from the try line; for try situations, defenders acquired a position $2-6 \mathrm{~m}$ from the try line).

In addition, vertical oscillations ( $z$ values) of defenders, which describe the up and down movements of players on each trial, were of greater magnitude when a try was scored than when a successful tackle was made. Differences in the range of vertical movements can be observed in Figure 4. For try situations, defenders produced a broader range of up and down movements while scoring tries, as is shown by the lowest $z$ value $(0.7 \mathrm{~m})$, than they did while making successful tackles (lowest $z$ value $=0.9 \mathrm{~m}$ ). The upper limit is the same for both situations. The results indicate that defenders decreased the distance to the ground in order to counterbalance attacker actions, and that there was a critical boundary distance to the ground below which defenders were not able to perform a successful tackle.

As is displayed in Figure 4A, when defenders advanced approximately $4 \mathrm{~m}$ into the field, vertical oscillations assumed values of $0.7-1.1 \mathrm{~m}$. For successful tackles (Figure 4B), defenders advanced in depth approximately $2 \mathrm{~m}$ and vertical oscillations assumed values of $0.9-1.1 \mathrm{~m}$. Additional analyses were performed to identify displacement curve shapes. For successful tackles, defender curves presented a better defined pattern - that is, advancement into the field and the abrupt decrease of $z$ values - suggesting an inverted " $J$ " shape. In contrast, when tries were scored, this displacement trajectory shape was not observed.

\section{Vertical Oscillation Analysis}

Vertical oscillations were identified by 3-D graphics. Figure 5 shows the means and $S D$ s of the $z$ values. The smaller $S D$ values indicate that vertical oscillations were more stable when successful tackles were made, which implies that defenders' actions were more stable under those circumstances. Moreover, for when a try was scored, one can observe an initial stability of $z$ values (until $1.6 \mathrm{sec}$ after initiation of the move) similar to that found for successful tackles (until $1.96 \mathrm{sec}$ after initiation of the move). Over time, Figure 5 shows enhanced variability of $z$ values when a try was scored (starting at approximately $2.12 \mathrm{sec}$ ) and a more abrupt change in the shape of the curve (at 4.72-6.28 sec), suggesting a phase transition. During successful tackles, there was also an increased variability (starting at approximately $1.96 \mathrm{sec}$ ) and a final decrease (at $4.84 \mathrm{sec}$ ), resulting in maintenance of the dyadic system's initial state of order.

\section{DISCUSSION}

Overall analysis of the data provided by the ANN revealed that this research method allowed us to collect data directly during reconstruction of a 3-D performance field, resulting in analysis of continuous time series data that represented interpersonal dynamics in a common subphase of rugby. In this discussion, we will follow an order of presentation in line with the goals we presented earlier, evaluate the strengths and weaknesses of the ANN, and conclude by identifying trends for future research.

To summarize, the first goal of our study was to identify phase transitions in a team ball sport with task constraints different from those of basketball. To achieve this goal, we used subphases of the team game of rugby. The data showed that the use of position-time graphics made it possible to identify phase transitions similar to those found by Araújo et al. (2002) in the sport of basketball. Our second goal was to present a 3-D analysis of interpersonal dynamics of attacker-defender dyads in rugby. Our findings show that the use of the ANN proved to be a reliable method for building a 3-D model of our performance field, which allowed us to analyze the trajectory of players from every angle selected. Finally, despite a quali- 

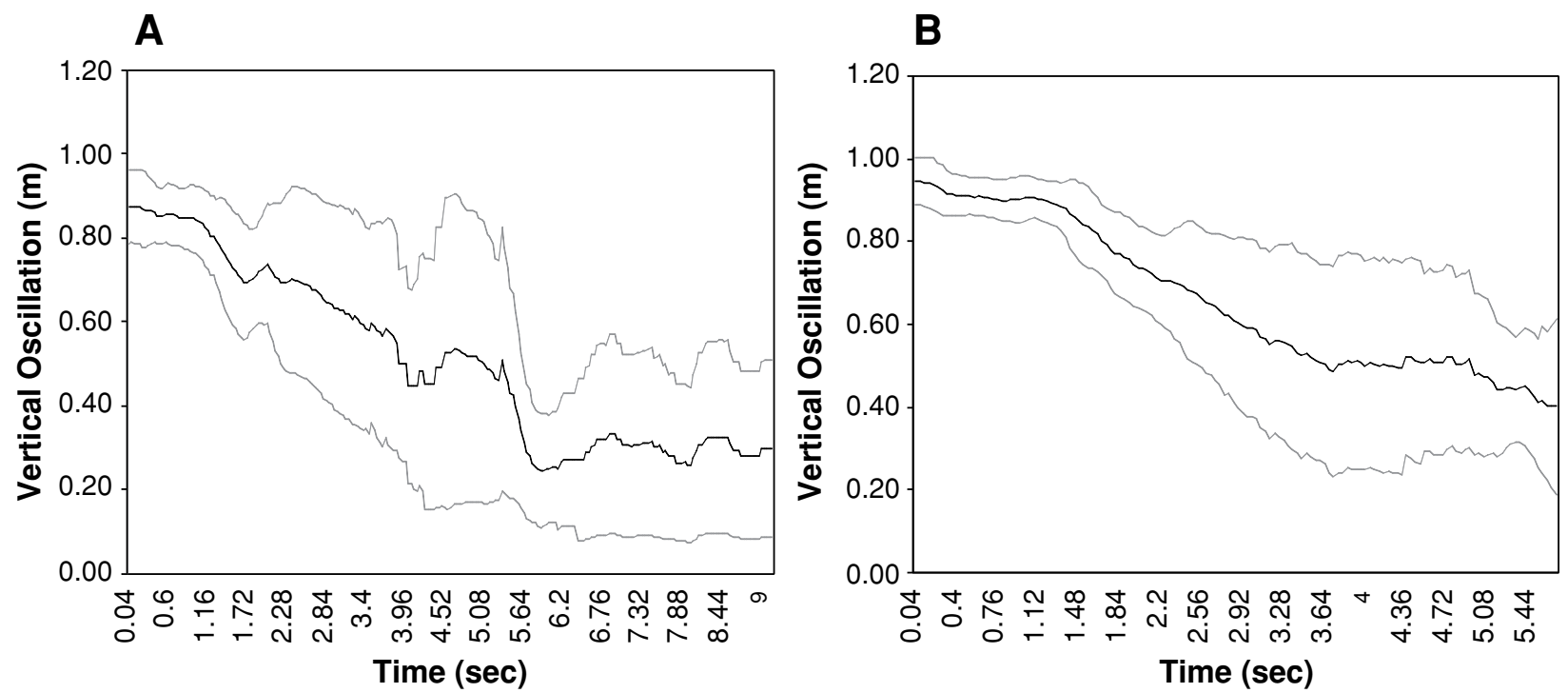

Figure 5. Mean (black line) and $S D$ s (gray lines) of defenders' vertical oscillations for tries (A) and successful tackles (B).

tative analysis of dynamical systems properties, there was a need to identify parameters to measure those properties, and the data appeared to reveal two parameters that might be key variables in a successful defense.

\section{Phase Transition Analysis}

Results revealed that the behavior of both players in a dyad emerged through exploration of the environment. Data indicated that decision making in team ball sports, exemplified in one-versus-one situations, can be characterized by a self-organized process depending on the specific ongoing interactions between the attacker and the defender in the dyad. Indeed, the results can be interpreted as displaying two key properties of a self-organized dynamical system: (1) maintenance of the initial state of order, with the advantage to the defender (Figure 3A); and (2) a phase transition, which occurs when the attacker gets past the defender (Figure 3B).

From an applied perspective, the results suggest that an ideal model of performance for both defender movement and attacker movement did not exist. Therefore, it is not desirable to determine in advance, during practice, the specific technical maneuvers that players should attempt to perform. Instead, the data suggest that to maintain goal-directed behavior, coaches should manipulate task constraints (e.g., by increasing or decreasing the field dimensions) to increase or decrease task difficulty, so that the players' performance is constrained by the specific demands of a particular performance situation.

\section{3-D Analysis}

Data confirmed that the ANN is a reliable tool for reconstructing a 3-D performance space, allowing a more complete analysis of sport performance.

Although previous research showed that dynamical systems properties of sport performance might be observed with a 2-D analysis, the present study shows that the key variables used to measure those properties could be more comprehensively identified with a 3-D analysis. More specifically, the symmetry-breaking process observed with position-time graphics (2-D) could be accurately measured by analysis of the vertical oscillations of a player's position, a variable that can be observed only with the use of 3-D analysis.

Differences found in the range of vertical movements revealed that a greater magnitude of vertical oscillations ( $z$ values) indicated more irregular up and down movements of defenders in attempts to prevent their opponents from passing them. In other words, the actions produced by the defender became more unpredictable, signifying that the defender was searching for a better position in order to attack his opponent. In addition, an increase in vertical oscillation variability revealed greater movement uncertainty due to exploratory activity to find a balanced position from which to tackle the attacker. Finally, a decrease in the stability of vertical oscillations signified that the defender was more sensitive to the perturbations induced by the attacker's actions.

The data appear to suggest that a balance between the forward motion and the vertical oscillation of the player may be key variables for the defender to consider when tackling in rugby. In addition, the inverted "J" shape of the displacement curves suggests that most of the defenders adopted a similar pattern of behavior, which led them to success.

To summarize, it can be concluded that successful defending in this subphase of rugby depends on the balance between advancing in the field and stabilizing the vertical oscillation. It is suggested that these two parameters might characterize what Greenwood (2003) termed "arriving in balance."

\section{Weaknesses and Strengths of ANNs}

Weaknesses. The first weakness of ANNs is that they are field specific, signifying that data cannot be imported 
from other performance fields because ANNs can run only data collected inside the specific reference structure used in training and validation procedures. Second, once we placed the cameras in specific locations, we could not move them to the right or the left to follow the action of a player or a group of players that might have interested us. In addition, we could not use the zoom lens to observe specific movements of players in greater detail. This technicality implies that the area designated to capture the motion of objects must be defined from the beginning. Every time cameras are moved, the calibration procedures must be repeated. Third, ANN training is a time-consuming and relatively slow process, owing to the iterative nature of backpropagation-based learning. The learning variables (e.g., learning rate, momentum coefficient) need to be adjusted according to the complexity of the problem. Too high a learning rate usually leads to poor prediction capabilities due to oscillation near the optimal set of internal weights, and, despite a rapid decrease in error (i.e., the difference between the ANN output and the actual values), they never converge (Basheer, 2000). Too low a learning rate is also undesirable due to the long time required for convergence to occur. The high and/or low definitions can be determined only during the network training process by observation of the evolution of error minimization. In that case, if the process is not suitable to solve a particular problem, it might be stopped and restarted after the learning variables are adjusted. Although several approaches to automatically varying the learning rate have been proposed, there are no general rules for establishing initial values for both the learning rate and the momentum coefficient. Nevertheless, the numbers of input and output neurons are defined by the problem of solving the network architecture (i.e., determining the number of neurons in the hidden layer[s] of the network) and are commonly established by a trial-and-error approach, since no rules for a priori establishment exist. This aspect is not exclusive for this kind of application (i.e., spatial coordinates calculation). Finally, there are other 3-D reconstruction approaches, such as the linear approaches (e.g., direct linear transformation), that require fewer reference points than ANNs do. However, as we stated before, to use these techniques we need to know the intrinsic parameters of the cameras, which significantly constrains the method of obtaining the data. Moreover, using linear models to analyze nonlinear phenomena can reduce the coherence between the theoretical and methodological approaches.

Strengths. First, despite the above-mentioned limitations, using ANNs as a method for analyzing sport performance brings practical advantages. Once the net for a specific performance field has been constructed, the only major concern is to locate the cameras at exactly the same place and height, to facilitate different types of movement analysis. Second, as we stated earlier, there is no need to know any of the intrinsic and/or extrinsic camera parameters, such as focal distance and camera position related to the origin. In addition, when a sufficient number of reference points are used, results produced by ANNs are accurate even in the presence of optical and/or digital distortion. Finally, as discussed earlier, to build the net, a set of points from a reference structure is needed for the training and validation procedures. Knowledge of certain kinds of measures that represent the depth, width, and height of the performance field (e.g., length of field lines, distance between lines, height of posts) can provide a set of points large enough to feed the net for those procedures. This method easily allows experimenters to collect images that are reliable for analyzing sport performance for any kind of field. Nowadays, naturalistic methods for studying decision making are gaining popularity (Klein, 2001), although there is little agreement on an adequate method for measuring behavior. In the present article, we propose that ANNs might be suitable methods for improving the analysis of decision making in sports tasks.

\section{Future Research}

From a qualitative point of view, it was possible to identify the existence of phase transitions in the sport of rugby. However, future research is needed to confirm these findings by identifying quantitative measures of this property. Further work is needed to simplify the use of ANNs for reconstructing a 3-D performance field in order to make them more user friendly. Finally, in this article we have suggested parameters that are related to single-player analysis, not to the properties of an attacker-defender dyad as a dynamical system. The need to identify a parameter (i.e., a collective variable) that could explain the behavior of an attacker-defender dyad remains a priority for future research.

\section{REFERENCES}

Araúuo, D., Davids, K., Bennett, S., Button, C., \& Chapman, G. (2004). Emergence of sport skills under constraints. In A. M. Williams \& N. J. Hodges (Eds.), Skill acquisition in sport: Research, theory and practice (pp. 409-433). London: Routledge.

Araújo, D., Davids, K., Saínhas, J., \& Fernandes, O. (2002). Emergent decision-making in sport: A constraints-led approach. In L. Toussaint \& P. Boulinguez (Eds.), International Congress of Movement, Attention \& Perception (p. 77). Poitiers, France: Université de Poitiers Press.

Bartlett, R. (1997). Introduction to sports biomechanics. London: Spon Press.

Basheer, M. H. (2000). Artificial neural networks: Fundamentals, computing, design, and application. Journal of Microbiological Methods, 43, 3-31.

Bates, B. T., Dufek, J. S., \& Davis, H. P. (1992). The effect of trial size on statistical power. Medicine \& Science in Sports \& Exercise, 24, 1059-1068.

Biscombe, T., \& Drewett, P. (1998). Rugby: Steps to success. Champaign, IL: Human Kinetics.

D'Appuzo, N. (2002), Surface measurement and tracking of human body parts from multi-image video sequences. ISPRS Journal of Photogrammetry \& Remote Sensing, 56, 360-375.

Fernandes, O., \& Caixinha, P. (2003, April). A new method in timemotion analysis in soccer training and competition. Paper presented at the Fifth World Congress of Science \& Football, Lisbon.

Greenwood, J. (2003). Total rugby: Fifteen-man rugby for coach and player. London: A\&C Black.

Gruen, A. (1997). Fundamentals of videogrammetry: A review. Human Movement Science, 16, 155-187.

HAYKIN, S. (1994). Neural networks: A comprehensive foundation. New York: Macmillan.

Hughes, M., \& Franks, I. M. (EDs.) (2004). Notational analysis of 
sport: Systems for better coaching and performance in sport (2nd ed.). London: Routledge.

Kelso, J. A. S. (1995). Dynamic patterns: The self-organization of brain and behaviour. Cambridge, MA: MIT Press.

Kelso, J. A. S., Buchanan, J. J., DeGuzman, G. C., \& Ding, M. (1993). Spontaneous recruitment and annihilation of degrees of freedom in biological coordination. Physics Letters A, 179, 364-371.

KLEIN, G. (2001). The fiction of optimization. In G. Gigerenzer \& R. Stelten (Eds.), Bounded rationality: The adaptive toolbox (pp. 103121). Cambridge, MA: MIT Press.

McGarry, T., \& Perl, J. (2004). Models of sports contests: Markov processes, dynamical systems and neural networks. In M. Hughes \& I. Franks (Eds.), Notational analysis of sport: Systems for better coaching and performance in sport (2nd. ed., pp. 227-242). London: Routledge.

Memon, Q., \& Khan, S. (2001). Camera calibration and three-dimensional world reconstruction of stereo-vision using neural networks. International Journal of Systems Science, 32, 1155-1159.

PerL, J. (2002). Game analysis and control by means of continuously learning networks. International Journal of Performance Analysis in Sport, 2, 21-35.

SMITH, L. (2001), An introduction to neural networks. Retrieved March 31,2004 , from University of Sterling, Department of Computing Sci- ence and Mathematics Web site: www.cs.stir.ac.uk/ 1ss/NNIntro/ InvSlides.html.

Stergiou, C., \& Siganos, D. (1996), Introduction to neural networks. Retrieved March 31, 2004, from www.doc.ic.ac.uk/ nd/surprise_96/ journal/vol4/cs11/report.html.

Zanone, P. G., \& Kelso, J. A. S. (1994). The coordination dynamics of learning. In S. [P.] Swinnen, H. Hever, J. Massion, \& P. Cassaer (Eds.) Interlimb coordination: Neural, dynamical, and cognitive constraints (pp. 462-490). San Diego: Academic Press.

\section{NOTE}

1. Due to different task constraints (e.g., the practice of tackling and the rule of 15 players per team), the time available to explore the dyadic context is likely to be shorter in rugby than in basketball, in which players could maintain interpersonal distances for longer periods. Because of the possibility of physical contact in rugby, different task constraints must be considered in interpersonal dynamics of rugby dyads than in those of basketball dyads.

(Manuscript received April 12, 2005; revision accepted for publication August 31, 2005.) 